\title{
Surface Chemistry and Extreme-Pressure Lubricant Properties of Dimethyl Disulfide
}

\author{
Javier Lara, ${ }^{\dagger}$ Thomas Blunt, $\$$ Peter Kotvis,, Alan Riga, ${ }^{\S}$ and Wilfred T. Tysoe ${ }^{* \dagger}$ \\ Department of Chemistry and Laboratory for Surface Studies, University of Wisconsin-Milwaukee, \\ Milwaukee, Wisconsin 53211; Benz Oil, Inc., 2724 Hampton Ave., Milwaukee, Wisconsin 53209; and \\ The Lubrizol Corporation, 29400 Lakeland Boulevard, Wickliffe, Ohio 44092
}

Received: November 18, 1997

\begin{abstract}
The growth kinetics of a film formed by the thermal decomposition of dimethyl disulfide on an iron foil are measured using a microbalance where the growth kinetics are parabolic (film thickness $X$ varies with time as $\left.X^{2} \propto t\right)$ at high reaction temperatures and pressures, indicating that it is limited by diffusion through the film. The activation energy for this process is $54.5 \pm 0.5 \mathrm{kcal} / \mathrm{mol}$. The growth rate becomes linear as the reaction temperature and/or reactant pressure is lowered, indicating that, under these circumstances, the reaction rate is limited by thermal decomposition of dimethyl disulfide at the growing interface. The activation energy for thermal decomposition at the interface is found to be $37.6 \pm 0.7 \mathrm{kcal} / \mathrm{mol}$, and a half-order kinetics pressure dependence for the surface reaction rate is found consistent with a reaction limited by the rate of dimethyl disulfide dissociation. Analysis of the resulting film using Raman and X-ray photoelectron spectroscopies as well as X-ray diffraction reveal the formation of FeS, which may be slightly nonstoichiometric. This film is similar to that formed by methanethiol, suggesting that they may both initially form a surface thiolate species that further reacts to form FeS. The half-order reaction kinetics noted above are consistent with this. Measurement of dimethyl disulfide as an extreme-pressure (antiseizure) additive reveals a plateau at an applied load of $\sim 4000 \mathrm{~N}$ in the seizure load versus additive concentration curve. It has previously been suggested that the plateau corresponds to the load at which the interface reaches the melting point of the solid lubricant layer (in this case proposed to be $\mathrm{FeS}$ ). Estimation of the interfacial temperature using a method developed previously yields an interfacial temperature of $\sim 1480 \mathrm{~K}$, in good agreement with the melting point of FeS.
\end{abstract}

\section{Introduction}

Extreme-pressure lubricants generally consist of several components dissolved in a hydrocarbon base fluid although, in some cases, water is used. ${ }^{1-6}$ The most important additive, from the point of view of the tribological properties of the lubricant, are antiseizure additives which help to prevent the lubricated surfaces from welding to each other while frictional work is being performed. An important class of such additives, which have hitherto been the focus of work that we have carried out, are chlorinated hydrocarbons. Unfortunately, these potentially have deleterious health and environmental effects. Despite this, these are still the most heavily used extreme-pressure (EP) additives since many of the environmental and health concerns have been addressed either by decreasing additive volatility and dispersibility or by recycling. Nevertheless, a considerable portion of the lubricant (generally estimated to be $\sim 20 \%$ ) is lost during manufacturing so that the goal of ultimately replacing these additives remains important.

From a fundamental point of view, the extreme-pressure regime is ideal for study since material is continually removed from the surface due to the high applied loads so that the contacting interfaces are no longer covered by oxide and carbonaceous materials that were likely to be there initially. Second, it has been shown that the interfacial temperature

* To whom correspondence should be addressed. Phone (414) 229-5222; FAX (414) 225-5036; E-mail wtt@csd.uwm.edu.

$\dagger$ University of Wisconsin-Milwaukee.

$\doteqdot$ Benz Oil, Inc.

$\S$ The Lubrizol Corporation. attained under these conditions can easily exceed $1000 \mathrm{~K},{ }^{7}$ so that the chemistry proceeding there is likely to be rather simple and the reaction kinetics rather fast under these conditions. It has, in fact, been suggested that the nature of the interface can simply be predicted using thermodynamic arguments, and this postulate has been borne out when using model chlorinated hydrocarbon extreme-pressure additives. ${ }^{8,9}$

In the case of chlorinated hydrocarbon additives, a model was proposed for their operation which suggested that an iron chloride plus carbon interfacial films that formed by thermal decomposition of the chlorinated hydrocarbon prevented seizure of the contacting surfaces, even under high applied loads. The steady-state thickness of the film during lubrication is a result of the rate at which it is reactively deposited by the additive thermally decomposing there and the rate at which it is worn from the surface. Assuming that the interface will seize when the layer is completely removed to reveal the bare metal below successfully predicts the variation in seizure load with additive concentration when using methylene chloride dissolved in a poly- $\alpha$-olefin as the model lubricating fluid. ${ }^{10-12}$ Suitable modifications of this model have allowed us to explain the EP properties of other additives such as chloroform ${ }^{13}$ and carbon tetrachloride. ${ }^{14}$

The work described below extends these investigations to examine the extreme-pressure properties of another important class of additives - those containing sulfur. There are a wide variety of these additives, but an important class of these consists 
of hydrocarbon molecules with sulfur linkages. Thus, molecules with $\mathrm{S}-\mathrm{S}$ linkages have been widely used as model sulfurcontaining additives, and the work here focuses on, perhaps the simplest example, dimethyl disulfide. A significant amount of work has already been carried out to examine the tribological properties of these types of additives. ${ }^{16-30}$ In addition, the surface chemistry of a wide range of sulfur-containing molecules has been examined on transition metals in order to try to understand the way in which they operate as hydrodesulfurization catalysts, and much of what has been learnt in the latter area will facilitate tribological understanding. ${ }^{31-36}$ Another area in which the chemistry of sulfur-containing molecules is important, in particular its reaction with iron, is to understand and ultimately prevent the corrosion of oil pipelines by the sulfur contaminants present in oil. ${ }^{30}$ This work has focused primarily on understanding the chemistry of thiols ( $\mathrm{RSH}$, where R is some hydrocarbon group). This, however, rapidly reacts with the surface to abstract the thiol hydrogen and deposit a thiolate on the surface. The subsequent chemistry is then that of the thiolates. When organic disulfides are used as lubricant additives, it has been proposed that they decompose via a $S-S$ bond cleavage to form similar thiolate species on the surface. ${ }^{16-22}$ It has also been demonstrated, in connection with understanding the corrosive behavior of methane thiol, that it ultimately reacts with iron to form a pyrrhotite $\left(\mathrm{Fe}_{1-x} \mathrm{~S}\right)$ film. ${ }^{30}$ A similar behavior is found here for the reaction of dimethyl disulfide, a not surprising result in view of the above remarks. It also appears that the reactively formed iron sulfide acts as an antiseizure film in the same way as $\mathrm{FeCl}_{2}$ does when chlorinated hydrocarbons are used as additives.

\section{Experimental Section}

Several pieces of experimental apparatus were used for these experiments, which have been described in detail elsewhere. ${ }^{8,9}$ Briefly, however, film growth experiments were carried out using a microbalance enclosed in a glass shroud that could be evacuated to $\sim 1 \times 10^{-7}$ Torr. The iron foil sample (Aesar, $99.99 \%$ ) was suspended in a quartz tube inserted into a tube furnace so that it could be heated. The dimethyl disulfide was transferred to a glass vial and purified by repeated freezepump-thaw cycles and introduced into the evacuated cell containing the foil and microbalance. The sample was then heated and the film growth rate measured from a change in mass of the sample as a function of time. The cell was fitted with a valve so that it could be evacuated and closed after reaction, keeping the sample for further Raman analysis without exposure to air (see below). Microbalance experiments have been used previously to measure the rate of formation of chloride films on iron from the thermal decomposition of chlorinated hydrocarbon vapors. ${ }^{14}$ In the latter case, it was found that film growth kinetics were apparently unaffected by precleaning the sample and merely heating in vacuo was sufficient to obtain reproducible results. This was not found to be the case with sulfurized hydrocarbons. In this case, it was necessary to pretreat the iron foil in oxygen $(100$ Torr, $620 \mathrm{~K}$ ) for $3600 \mathrm{~s}$ and then reduce it in hydrogen (200 Torr, $900 \mathrm{~K}$ ) for $7200 \mathrm{~s}$. Subsequent kinetic experiments then yielded reproducible results. The resulting films were analyzed using an array of techniques including Raman spectroscopy, X-ray photoelectron spectroscopy, and $\mathrm{X}$-ray diffraction.

$\mathrm{X}$-ray photoelectron spectroscopic data were collected using a VG Escalab spectrometer equipped with a fast entry interlock. Spectra were collected using unmonochromated $\mathrm{Mg} \mathrm{K} \alpha$ radia-

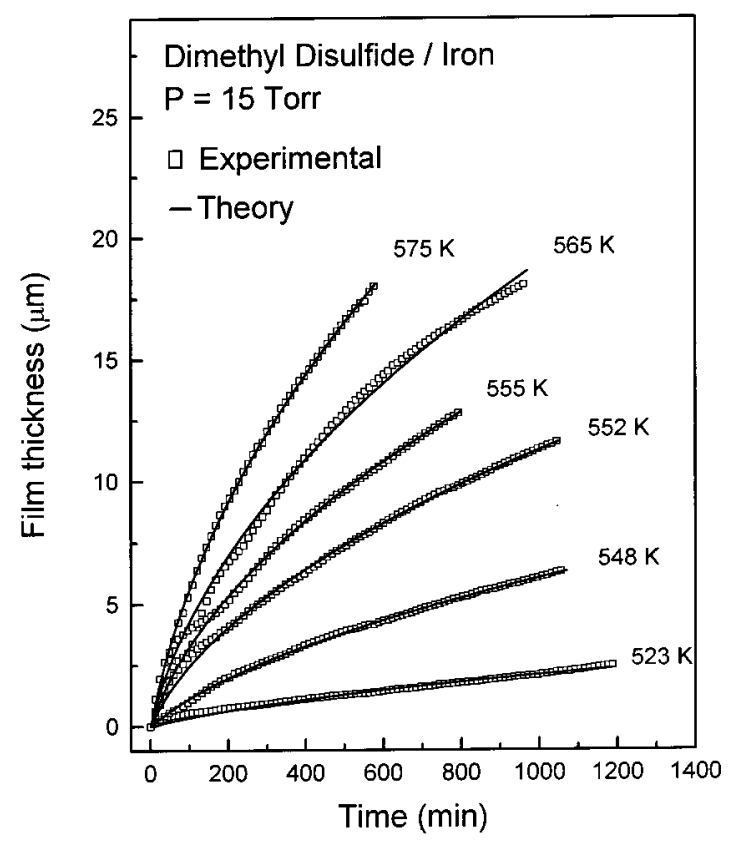

Figure 1. Plot of film thickness versus time for the reaction of 15 Torr of dimethyl disulfide with iron foil as a function of temperature ( $\square)$. Reaction temperatures are indicated adjacent to the corresponding curve. Solid lines represent theoretical fits to the experimental data.

tion and the photoemitted electrons analyzed using a $100 \mathrm{~mm}$ radius hemispherical analyzer operating with a pass energy of $50 \mathrm{eV}$. This yielded an overall spectral resolution of $0.6 \mathrm{~V}$.

Raman spectra were collected using $5145 \AA$ excitation radiation from an argon ion laser operating in the backscattering mode. Films, which were not exposed to air, were used for this purpose. Since the signals from the films were rather small, plasma lines were removed by diverting the light through two prisms and then passing it through an iris. The scattered radiation was analyzed using a Spex double monochromator, and the photons were detected using a Peltier-effect-cooled photomultiplier.

A Phillips X-ray diffraction unit was used to identify the crystalline components in the samples. These were collected using a copper target emitting X-rays with wavelengths of $1.5405 \AA$ at a fixed divergence of $1^{\circ}$ and detection slits set at $0.1^{\circ}$. The X-ray excitation energy was $40 \mathrm{kV}$ at $50 \mathrm{~mA}$ emission. The $2 \theta$ range was from $20^{\circ}$ to $100^{\circ}$ at a scan speed of $0.02 \mathrm{deg} / \mathrm{s}$.

Tribological measurements were made on a model lubricant synthesized by dissolving various amounts of dimethyl disulfide in a poly- $\alpha$-olefin base oil. The model lubricant was tested using a conventional pin and V-block apparatus where the seizure load is recorded as a function of additive concentration. ${ }^{12-15}$ Seizure loads are measured by monitoring the torque required to rotate the pin at a constant angular velocity (290 rpm). The torque increases linearly with load, and the slope of the plot of torque as a function of load is proportional to the interfacial coefficient of friction. However, at a certain critical load, the torque required to rotate the pin suddenly increases, and this load is recorded as the seizure load.

\section{Results}

Shown in Figure 1 are the result of film growth experiments using 15 Torr of dimethyl disulfide as a function of growth temperature. As noted above, it was found necessary to stringently clean the iron sample prior to reaction to remove 


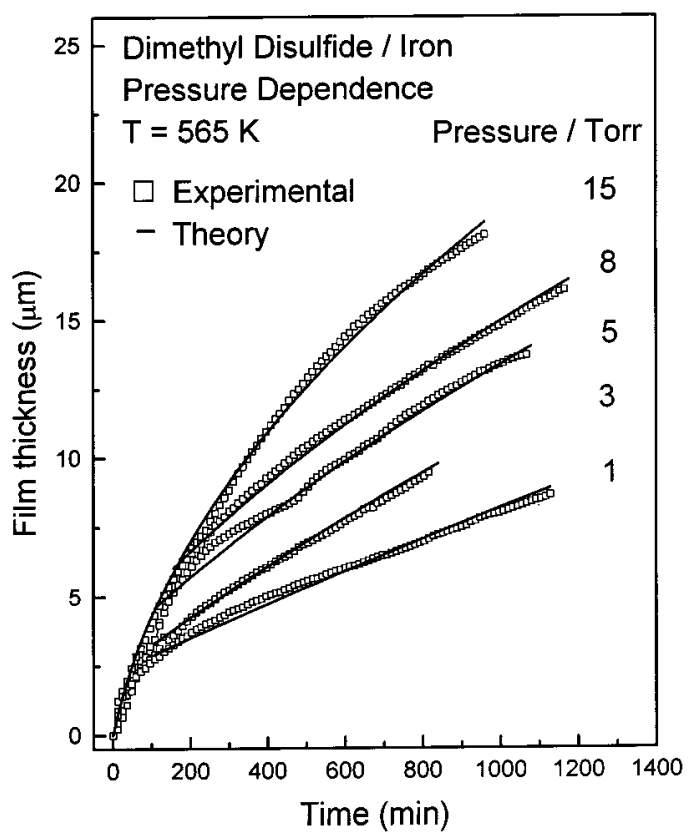

Figure 2. Plot of film thickness versus time for the reaction of dimethyl disulfide with iron foil at $565 \mathrm{~K}$ as a function of gas pressure ( $\square$ ). Reactant pressures are indicated adjacent to the corresponding curve. Solid lines represent theoretical fits to the experimental data.

surface contaminants. This procedure consisted of heating at $620 \mathrm{~K}$ in 100 Torr of oxygen to remove any organic or carbonaceous deposits. The oxygen was then removed from the cell enclosing the sample and microbalance, and the sample was heated in 200 Torr of hydrogen at $900 \mathrm{~K}$ to reduce any oxide formed at the surface to yield a clean iron foil. This procedure produced samples that yielded reproducible film growth kinetics and was repeated for each foil sample. The change in mass of the sample was converted using a prescription described elsewhere using the density of the film formed on the surface. ${ }^{12-15}$ As shown below, the surface consists of FeS, and thicknesses were measured assuming that the film consists entirely of this material. Films up to $\sim 20 \mu \mathrm{m}$ in thickness are formed, and the shape of the curve for films grown at $575 \mathrm{~K}$ is parabolic where the film thickness $X$ varies with time as $X^{2} \propto$ $t$. This has been shown to be due to a growth mechanism in which the kinetics are limited by transport through the film. ${ }^{37-42}$ At lower temperatures $(523 \mathrm{~K})$, the growth curve is essentially linear with time, suggesting that film growth is limited by the thermal decomposition of dimethyl disulfide at the surface. Note that these growth modes are essentially identical to those found for the thermal decomposition of $\mathrm{CH}_{3} \mathrm{SH}$ on iron. ${ }^{30}$

The corresponding pressure dependence is shown in Figure 2 for reaction at $565 \mathrm{~K}$ where now the reactant pressure is varied between 1 and 15 Torr. Again, mass changes were converted to film thickness assuming a film formed entirely of FeS. There is an initial rapid growth rate for $\sim 100 \mathrm{~min}$ where a film $\sim 2.5$ $\mu \mathrm{m}$ thick is formed, and then the film grows more slowly thereafter. The growth is essentially linear at low pressures and changes to parabolic as the reactant pressure increases. This is in accord with the notion that the linear regime is reaction rate limited, and as the surface reaction is accelerated by the addition of more reactant, it becomes limited instead by the rate of film formation. Similar behavior has been seen for the thermal decomposition of carbon tetrachloride on iron, and this situation has been analyzed theoretically. Similar theoretical fits are made here, and the solid lines plotted onto the kinetic data of Figures

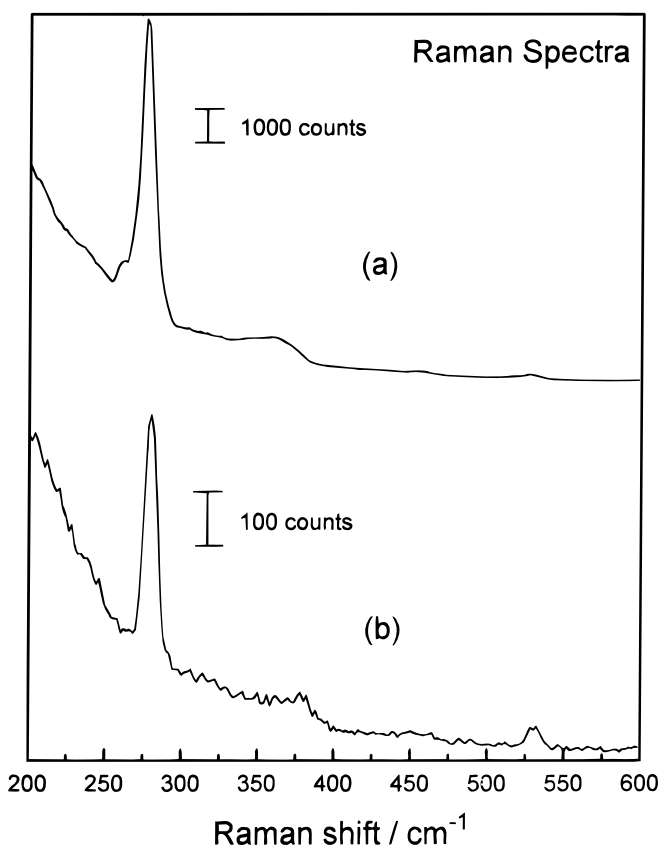

Figure 3. Raman spectra of (a) FeS powder and (b) a film grown on iron foil by the thermal decomposition of 15 Torr of dimethyl disulfide at $625 \mathrm{~K}$.

1 and 2 are the results of these fits and will be discussed in greater detail below.

The Raman spectrum of a typical film grown at $625 \mathrm{~K}$ using 15 Torr of dimethyl disulfide is shown in Figure $3 \mathrm{~b}$. This has a prominent feature at $\sim 280 \mathrm{~cm}^{-1}$, with weaker, but welldefined peaks at $\sim 380$ and $530 \mathrm{~cm}^{-1}$. A comparison fingerprint spectrum for FeS is shown in Figure 3a. The spectrum for FeS (Figure 3a) exhibits a similar sharp, intense peak at $280 \mathrm{~cm}^{-1}$ with weaker features at 360 and $530 \mathrm{~cm}^{-1}$ and so is essentially identical to the spectrum of the reactively formed film. This does not agree with the only Raman of FeS spectrum previously published which shows a single peak at $472 \mathrm{~cm}^{-1} .43$ Note that the Raman spectrum of elemental sulfur exhibits strong peaks at 150,220 , and $470 \mathrm{~cm}^{-1} \cdot 44,45$ This suggests that the feature assigned to FeS $\left(472 \mathrm{~cm}^{-143}\right)$ is in fact due to sulfur. Note that in our spectrum there are no features that correspond to elemental sulfur. The Raman spectrum of pyrite $\left(\mathrm{FeS}_{2}\right)$ comprises three features at 340, 380, and $415 \mathrm{~cm}^{-1},{ }^{44,46}$ suggesting that there is no $\mathrm{FeS}_{2}$ in our sample. It should be emphasized that the Raman spectra of films were measured in situ without intervening air exposure. This is particularly important since $\mathrm{FeS}_{2}$ oxidizes rather easily in air ${ }^{47,48}$ and has a Raman spectrum with its most predominant features at 150, 220, and $473 \mathrm{~cm}^{-145}$ due to elemental sulfur, and where the $\mathrm{FeS}_{2}$ modes at 340 and $380 \mathrm{~cm}^{-1}$ are much weaker. Spectra of $\mathrm{FeS}_{2}$ collected with our spectrometer corroborate these assignments.

The corresponding S $2 p$ X-ray photoelectron peaks for films grown at various temperatures are shown in Figure 4. In all cases, the S $2 p$ binding energy is $161.5 \pm 0.2 \mathrm{eV}$. The asymmetry of the peak is due to the unresolved spin-orbit coupling split doublet. The $S 2 p$ binding energy is indicative of the type of sulfide present since FeS has a binding energy of $161.5 \mathrm{eV}$ whereas in $\mathrm{FeS}_{2}$ the binding energy shifts to 162.2 eV. ${ }^{49-51}$ Thus, X-ray photoelectron spectroscopy also indicates the presence of FeS.

Finally, the X-ray diffraction pattern of the inorganic film formed on the iron substrate is shown in Figure 5 and is typically due to a nonstoichiometric, oriented FeS. The peak positions were compared with standards, ${ }^{52}$ and all of the peaks observed 


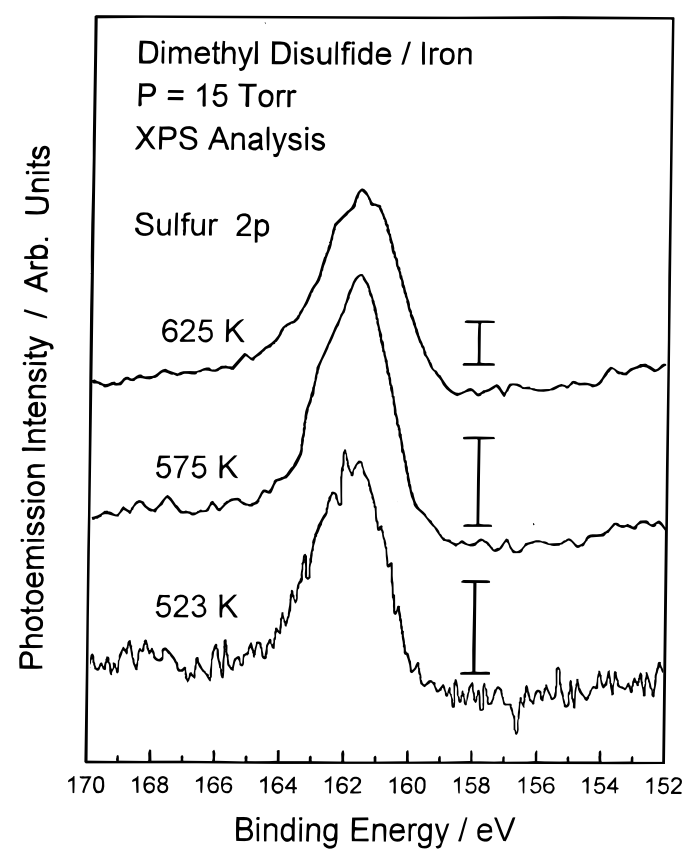

Figure 4. Sulfur $2 p$ X-ray photoelectron spectra of films grown by the thermal decomposition of 15 Torr of dimethyl disulfide grown at 523,575 , and $625 \mathrm{~K}$. The temperatures at which the films were grown is indicated adjacent to each spectrum, and the vertical lines indicates a count rate of $1 \mathrm{kHz}$ for each spectrum.

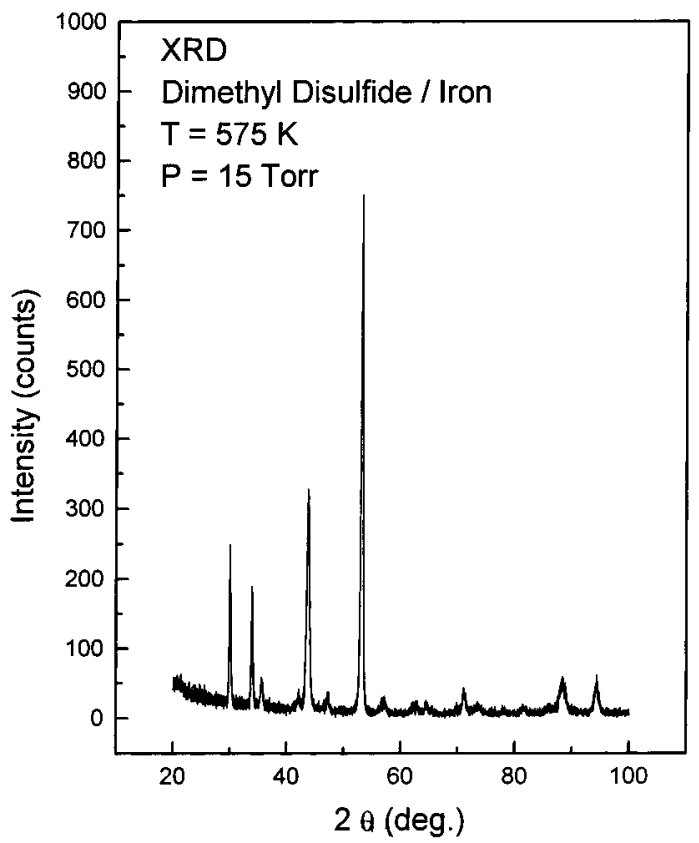

Figure 5. X-ray diffraction patterns of films grown by the thermal decomposition of 15 Torr of dimethyl disulfide at $575 \mathrm{~K}$ on iron.

correspond well to the pattern of FeS except that the $1.72 \AA$ $\left(2 \Theta=53.19^{\circ}\right)$ line is more intense than indicated in the standard patterns, suggesting that the layer may be oriented.

Finally, the tribological properties of dimethyl disulfide as an antiseizure additive under extreme-pressure conditions are displayed in Figure 6 in the form of a plot of seizure load versus the sulfur concentration of the model lubricant additive in weight percent. The seizure load increases with the addition of sulfur to the base oil (poly- $\alpha$-olefin) consistent with its proposed properties as an antiseizure additive and shows the formation of a plateau at $\sim 6 \mathrm{wt} \%$ added sulfur at a load of $\sim 4000 \mathrm{~N}$. As the concentration of additive is increased to $8 \mathrm{wt} \%$, however,

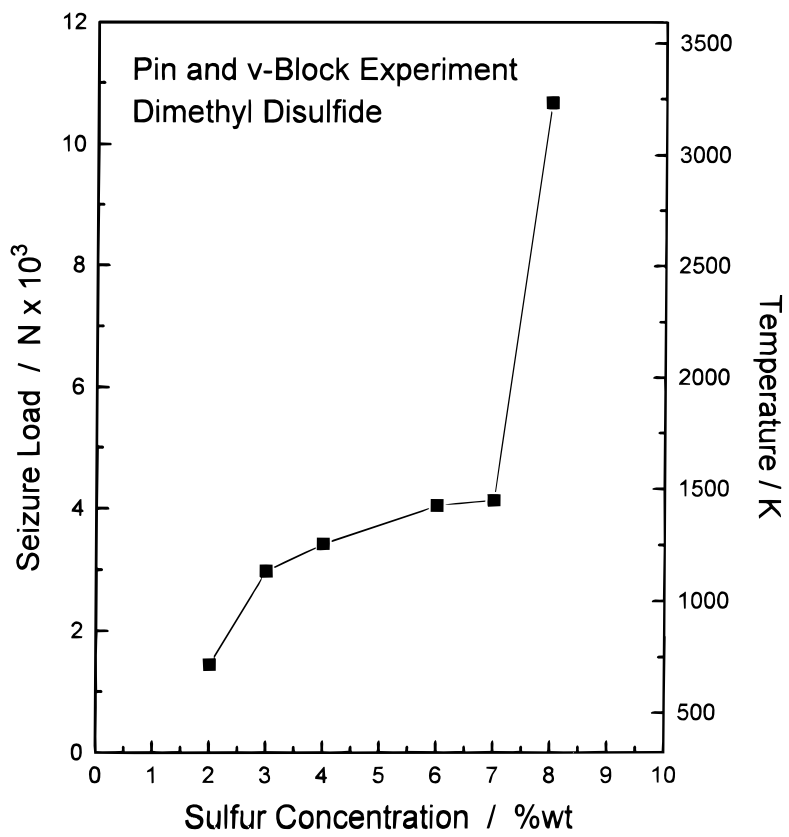

Figure 6. Plot of seizure load versus additive concentration expressed as wt $\% \mathrm{~S}$ when using dimethyl disulfide dissolved in poly- $\alpha$-olefin as a model lubricant using the pin and V-block apparatus. Shown on the right ordinate is the interfacial temperature calibrated using the interfacial coefficient of friction also measured with the pin and V-block apparatus (see text).

the antiseizure ability improves drastically, and the lubricant is now able to sustain loads of at least $11000 \mathrm{~N}$ before seizure. This type of behavior, i.e., the existence of a plateau, has been noted previously when using $\mathrm{CHCl}_{3}$ as an additive. ${ }^{53}$ The coefficient of friction was also measured from the torque versus load data after $60 \mathrm{~s}$ equilibration at each load when $6 \mathrm{wt} \%$ of $\mathrm{S}$ additive from dimethyl disulfide was present and yielded a value of $0.12 \pm 0.01$

\section{Discussion}

Nature of the Reactively Formed Film and Analysis of Their Growth Kinetics. Raman and X-ray photoelectron spectroscopies as well as X-ray diffraction data all indicate the formation of an FeS layer. A similar film has been found from the thermal decomposition of methane thiol on iron, where the film was also examined using scanning electron microscopy and $\mathrm{X}$-ray diffraction. In this case, the formation of hexagonal $\mathrm{Fe}_{1-x} \mathrm{~S}$ is observed. SEM images show the formation of an initial fine-grain equiaxed layer followed by long columnar grains growing with the $c$ axis approximately normal to the substrate. The kinetics of sulfidation is linear in the range 533$633 \mathrm{~K}$ and parabolic in the range $643-813 \mathrm{~K}$. The apparent activation energy of the parabolic process is estimated to be $30.2 \pm 2.2 \mathrm{kcal} / \mathrm{mol} .{ }^{30}$

It has been shown previously for the growth of $\mathrm{FeCl}_{2}$ films from the thermal decomposition of $\mathrm{CCl}_{4}$ that both parabolic and linear growth regimes are detected, and the film thickness $X$ as a function of time $t$ is given by

$$
X^{2}+B\left(P_{\mathrm{r}}, T\right) X-A(T) t=0
$$

where $B=4 D_{i} l /\left(k_{\mathrm{r}} P_{\mathrm{r}}^{n}\right)$ and $A=4 D_{i} l / \Omega$, where $P_{\mathrm{r}}$ is the reactant pressure, $n$ the reaction order, and $k_{\mathrm{r}}$ the reaction rate constant. ${ }^{14,15} D_{i}$ is the growth diffusion coefficient and $l$ the jump distance in the growing film, and $\Omega$ is the volume of film formed by the decomposition of a reactant molecule. Assuming that $n$ 
Temperature / K

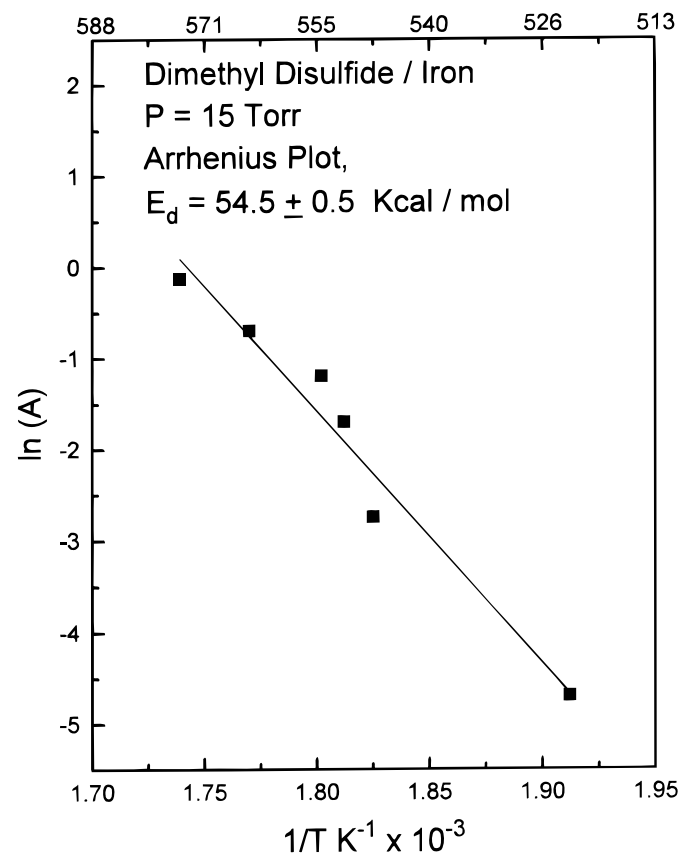

Figure 7. Arrhenius plot of $\ln (A)$ versus $1 / T$ (see text) for the thermal decomposition of 15 Torr of dimethyl disulfide on iron. This yields an activation energy for diffusion through the film of $54.6 \pm 0.4 \mathrm{kcal} /$ mol.

is a positive number, it is clear that at high pressures the growth kinetics are parabolic and revert to linear growth as the pressure is lowered as observed experimentally. This equation was fit to the growth data displayed in Figures 1 and 2, and the solid lines in these curves are then the result of these least-squares fits; and clearly the fit to the experimental data is good. The parameter $A$ depends on the activation energy for ion diffusion through the growing FeS film, and an Arrhenius plot for this value is displayed in Figure 7. This yields a good straight line where the activation energy for diffusion is given by $54.6 \pm$ $0.4 \mathrm{kcal} / \mathrm{mol}$. This value is somewhat higher than that found for film growth from methanethiol. ${ }^{30}$ The temperature dependence for the parameter $B$ is also plotted in Arrhenius form in Figure 8 and yields a value of $17.0 \pm 0.1 \mathrm{kcal} / \mathrm{mol}$. This value corresponds to the energy difference between the diffusion and reaction activation energies and accounts for the change from linear to parabolic growth as the reaction temperature changes. Thus, $E_{\mathrm{d}}-E_{\mathrm{k}}=17.0 \pm 0.1 \mathrm{kcal} / \mathrm{mol}$ where $E_{\mathrm{d}}$ is the activation energy for ion diffusion (measured as $54.6 \pm 0.4 \mathrm{kcal} / \mathrm{mol}$ above) and $E_{\mathrm{k}}$ is the activation energy for the rate-limiting reaction step. This gives a value for the reaction activation energy as $37.6 \pm 0.7 \mathrm{kcal} / \mathrm{mol}$. The pressure-dependent data shown in Figure 9 provide insights into the nature of the ratelimiting step. Here $\ln (B)$ is plotted versus $\ln (P)$ where $P$ is the pressure of dimethyl disulfide and yields a slope of $-0.6 \pm$ 0.1 . The formula for $B$ given above depends on $P^{-n}$ where $n$ is the reaction order and clearly yields $n \sim 0.5$. The rate-limiting step for reactive film formation is of $\sim 0.5$ order in dimethyl disulfide pressure which suggests that the rate-limiting step is a dissociation process. Since the films formed from methanethiol strongly resemble the films grown in this case, this step is likely to be the dissociative adsorption of dimethyl disulfide to yield thiolate species, a common intermediate when using both dimethyl disulfide and methanethiol. The subsequent fate of this species during film growth is not known although, since methane is generally found to desorb from metal surfaces in ultrahigh vacuum, ${ }^{54-60}$ clearly a portion of these species must

\section{Temperature / $\mathrm{K}$}

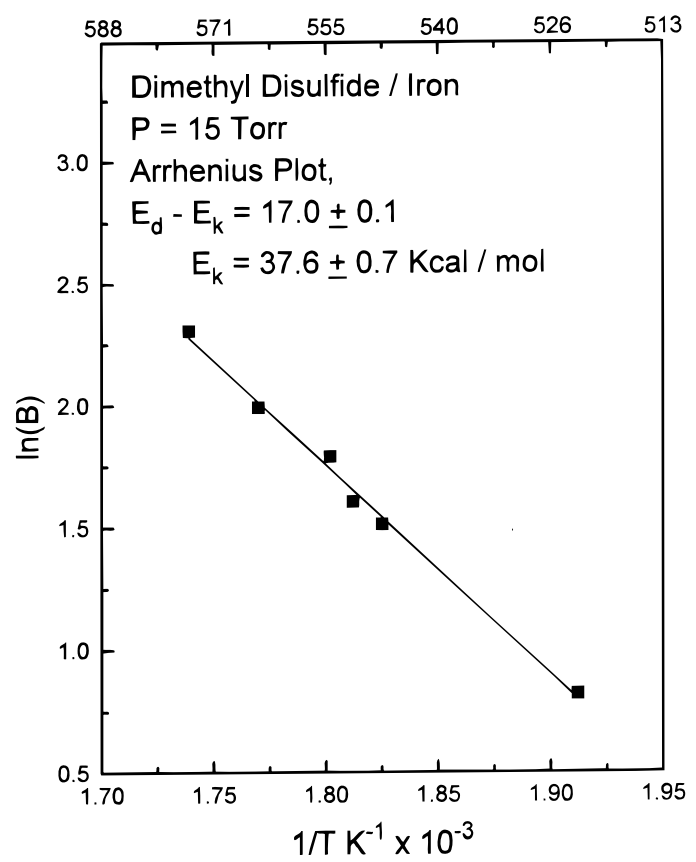

Figure 8. Arrhenius plot of $\ln (B)$ versus $1 / T$ (see text) for the thermal decomposition of 15 Torr of dimethyl disulfide on iron. This yields an activation energy of $17.0 \pm 0.1 \mathrm{kcal} / \mathrm{mol}$ for the difference in activation energies for diffusion and surface decomposition (see text).

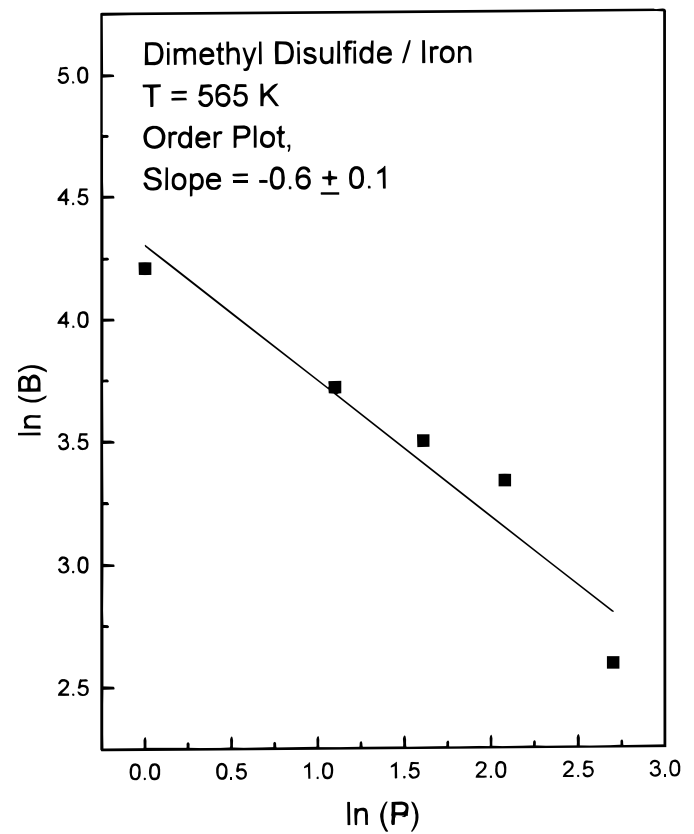

Figure 9. Plot of $\ln (B)$ versus $\ln (P)$ (see text) where $P$ is the pressure of dimethyl disulfide reacting on iron foil at $565 \mathrm{~K}$. This yields a surface reaction dimethyl disulfide pressure dependence of $0.6 \pm 0.1$.

further thermally decompose to yield carbon and sulfur, and the sulfur then oxidizes the iron substrate to form FeS. Molecular beam experiments on clean iron substrates in ultrahigh vacuum will further serve to clarify this. Note that previous works on the chemistry of disulfides have proposed the formation of elemental sulfur and monosulfides via thiosulfoxide intermediates and further reaction of the elemental sulfur with iron to form iron sulfide ${ }^{28,29}$ and/or the formation of three-electron-bonded radical anions with exoemitted electrons from frictional surfaces which dissociate practically 
instantaneously to free thiol radicals and thiolate species and further formation of iron sulfide. ${ }^{29}$

In the case of films formed from chlorinated hydrocarbons, the carbon resulting from this reaction often agglomerates to form small carbonaceous particles. ${ }^{12}$ These are, however, not detected here. Carbon was detected in X-ray photoelectron spectroscopy although it is not clear whether this arises from contamination because of exposure of the samples to air or was deposited during the reaction. Clearly, however, it is likely that some carbon will remain on the surface but that this is insufficient to coalesce into particles detectable in Raman spectroscopy.

Tribological Behavior of Dimethyl Disulfide. The tribological properties of dimethyl disulfide under extreme-pressure conditions are displayed in Figure 6. These results show the formation of a plateau and a sudden increase in load for the addition of more that $7 \mathrm{wt} \% \mathrm{~S}$ from dimethyl disulfide. The interfacial coefficient of friction during these experiments is 0.12 \pm 0.02 . It has been shown previously that the interfacial temperature in the pin and v-block apparatus varies linearly with applied load as

$$
T=T_{0}+\alpha L
$$

where $T_{0}$ is the ambient temperature $(322 \mathrm{~K})$ and $\alpha$ is a constant that is given by $2.27 \mu \mathrm{K} / \mathrm{N}$ where $\mu$ is the interfacial coefficient of friction. Substituting the value measured here yields a value of $\alpha=0.272 \mathrm{~K} / \mathrm{N}$, and the temperature scale marked on the right ordinate is calculated using this formula. It is clear that extremely high temperatures up to $2500 \mathrm{~K}$ can be attained during these experiments in the pin and V-block apparatus, when dimethyl disulfide is used as an additive, and according to the results presented in the previous section, the dimethyl disulfide additive should thermally decompose rather rapidly to yield $\mathrm{FeS}$ and perhaps incorporate some carbon. A model has been proposed for the operation of chlorinated hydrocarbons as additive in which the film formed by their thermal decomposition $\left(\mathrm{FeCl}_{2}\right.$ in this case) is present during lubrication and that the equilibrium film thickness is a balance between the rate of formation by chemical reaction and its wear rate. It is further assumed that seizure occurs when the film thickness diminishes to zero. A similar proposal is made in this case where it is assumed that the reactively formed FeS film acts to prevent seizure. At the high interfacial temperatures present during extreme-pressure lubrication, the wear rate becomes infinite as the interfacial temperature reaches the melting point of the antiseizure film. When chlorinated hydrocarbons are used, this leads to a plateau seizure load which yields an interfacial temperature corresponding to the melting point of $\mathrm{FeCl}_{2}(\sim 940$ $\left.\mathrm{K}^{61}\right)$. In the case of the FeS film, this model would then predict that the interfacial temperature at the plateau should be the melting point of $\mathrm{FeS}\left(\sim 1460 \mathrm{~K}^{61}\right)$, and the scale shown in Figure 6 agrees well with this value.

The seizure load corresponds to temperatures that significantly exceed this value when the sulfur concentration is greater than $7 \mathrm{wt} \%$, indicating that some other, higher melting point material prevents seizure under these conditions. As noted above, this effect has been seen previously with chloroform ${ }^{53}$ and carbon tetrachloride ${ }^{15}$ and was ascribed to the formation of a carbide and/or graphitic film in those cases. A similar assignment is tentatively made here, although further work needs to be done to verify this.

\section{Conclusion}

Dimethyl disulfide thermally decomposes on an iron surface via half-order kinetics to yield a film that consists of FeS. This kinetic behavior suggests the dissociative formation of thiolate species, and the activation energy for this process at the growing $\mathrm{FeS}$ interface is $\sim 37.6 \mathrm{kcal} / \mathrm{mol}$. Dimethyl disulfide also acts as an antiseizure extreme-pressure additive, and in this case, the seizure load increases with additive concentration until it reaches a plateau. This behavior has been found previously, and the interfacial temperature at the plateau has been shown to correspond to the melting point of the interfacial film. A similar phenomenon is found in this case since the interfacial temperature at the plateau is $\sim 1460 \mathrm{~K}$, in agreement with the melting point of FeS. Finally, a drastic increase in seizure load is found for sulfur concentrations greater than $\sim 7 \mathrm{wt} \%$, and this is ascribed to the formation of a high melting point carboncontaining film.

Acknowledgment. We gratefully acknowledge support of this work by the Chemistry Division of the National Science Foundation under Grant CHE-9213988.

\section{References and Notes}

(1) Tabor, D. J. Lubr. Technol. 1981, 103, 169

(2) Nakai, H.; Kanda, N. Proc. JSLE Int. Trib. Conf. 1985, 2, 515

(3) Mould, R. W.; Silver, H. B.; Syrett, R. J. Wear 1973, 22, 269.

(4) Dorinson, A. ASLE Trans. 1973, 16, 22.

(5) Mould, R. W.; Silver, H. B.; Syrett, R. J. Wear 1973, 26, 27.

(6) Clason, D. L. Lubr. Sci. 1989, 1, 281.

(7) Lara, J.; Kotvis, P. V.; Tysoe, W. T. Trib. Lett. 1997, 3, 303

(8) Blunt, T. J.; Kotvis, P. V.; Tysoe, W. T. Trib. Trans., in press.

(9) Blunt, T. J.; Kotvis, P. V.; Tysoe, W. T. Trib. Trans., in press.

(10) Kotvis, P. V.; James, M. N.; Tysoe, W. T. Wear 1992, 153, 305.

(11) Kotvis, P. V.; Huezo, L.; Millman, W. S.; Tysoe, W. T. Applications of Surface Science and Advances in Tribology: Experimental Approaches; ACS Symp. Ser. 485; American Chemical Society: Washington, DC, 1992.

(12) Kotvis, P. V.; Huezo, L. A.; Tysoe, W. T. Langmuir 1993, 9, 467

(13) Huezo, L.; Kotvis, P. V.; Crumer, C.; Soto, C.; Tysoe, W. T. Appl. Surf. Sci. 1994, 78, 113.

(14) Lara, J.; Molero, H.; Ramirez-Cuesta, A.; Tysoe, W. T. Langmuir 1996, 12,2488 . 10.

(16) Davey, W.; Edwards, E. D. Wear 1957, 1, 291.

(17) Forbes, E. S.; Reid, A. J. D. ASLE Trans. 1972, 16, 50.

(18) Forbes, E. S. Wear 1970, 15, 87.

(19) Allum, K. G.; Forbes, E. S. J. Inst. Petrol. 1967, 53, 173.

(20) Prutton, C. F.; Turnbull, D.; Dlouhy, G. J. Inst. Petrol. 1946, 32, 96.

(21) Davey, W. J. Inst. Petrol. 1946, 32, 575.

(22) Spikes, H. A.; Cameron, A. ASLE Trans. 1973, 17, 283.

(23) Bovington, C. H.; Dacre, B. ASLE Trans. 1982, 25, 44.

(24) Dacre, B.; Bovington, C. H. ASLE Trans. 1982, 25, 272

(25) Tomaru, M.; Hironaka, S.; Sakurai, T. Wear 1977, 41, 141.

(26) Murakami, T.; Sakai, T.; Yamamoto, Y.; Hirano, F. ASLE Trans. 1985, 28, 363 .

(27) Plaza, S.; Mazurkiewicz, B.; Gruzinski, R. Wear 1994, 174, 209

(28) Plaza, S. ASLE Trans. 1987, 30, 493.

(29) Kajdas, C. ASLE Trans. 1985, 28, 21.

(30) Pareek, V. K.; Ramanarayanan, T. A.; Mumford, J. D.; Ozekcin,

A.; Scanlon, J. C. Oxid. Met. 1994, 41, 323.

(31) Roberts, J. T.; Friend, C. M. J. Phys. Chem. 1988, 92, 5205.

(32) Roberts, J. T.; Friend, C. M. Surf. Sci. 1988, 198, L321.

(33) Roberts, J. T.; Friend, C. M. J. Am. Chem. Soc. 1987, 109, 3872.

(34) Roberts, J. T.; Friend, C. M. J. Am. Chem. Soc. 1986, 108, 7204.

(35) Heise, W. H.; Tatarchuk, B. Surf. Sci. 1989, 207, 297.

(36) Parker, B.; Gellman, A. J. Am. Chem. Soc. 1993, 292, 223

(37) Cabrera, N.; Mott, N. F. Rep. Prog. Phys. 1949, 12, 163

(38) Hauffe, K. Oxidation of Metals; Plenum Press: New York, 1965

(39) Kofstad, P. High-Temperature Oxidation of Metals; John Wiley and Sons: New York, 1966.

(40) Evans, E.; Norfolk, D. J.; Swan, T. J. Electrochem. Soc. 1978, 125 1180

(41) Waber, J. T. J. Chem. Phys. 1952, $20,734$.

(42) Anderson, S. Phys. Rev. 1946, 69, 52

(43) Park, H. L.; White, W. B. Phys. Status Solidi 1987, K69, 144.

(44) Turcotte, S. B.; Benner, R. E.; Riley, A. M.; Li, J.; Wadsworth, M. E. Appl. Opt. 1993, 32, 935.

(45) Mycroft, J. R.; Bancroft, G. M.; McIntyre, N. S.; Lorimer, J. W.; Hill, I. R. J. Electroanal. Chem. 1990, 292, 139. 
(46) Vogt, H.; Chattopadhyay, T.; Stolz, H. J. J. Phys. Chem. Solids 1983, 44, 869 .

(47) Hamilton, I. C.; Woods, R. Aust. J. Chem. 1978, 118, 327.

(48) Michell, D.; Woods, R. Aust. J. Chem. 1978, 31, 27.

(49) Pratt, A. R.; Muir, I. J.; Nesbitt, H. W. Geochim. Cosmochim. Acta 1994, $58,827$.

(50) Mycroft, J. R.; Nesbitt, H. W.; Pratt, A. R. Geochim. Cosmochim. Acta 1995, 59, 721 .

(51) Buckley, A. N.; Woods, R. Appl. Surf. Sci. 1985, 22/23, 280.

(52) International Crystallographic Diffraction Database, cards 22-0358 and $20-0535$.

(53) Tysoe, W. T.; Surerus, K.; Lara, J.; Blunt, T. J.; Kotvis, P. V. Trib. Lett. 1995, 1, 39 .
(54) Koestner, R. J. Stohr, J.; Gland, J. L.; Kollin, E. B.; Sette, F. Chem Phys. Lett. 1985, 120, 285

(55) Rufael, T. S.; Koestner, R. J.; Killin, E. B.; Salmeron, M.; Gland, J. Surf. Sci. 1993, 297, 272.

(56) Sexton, B. A.; Nyberg, G. L. Surf. Sci. 1986, 165, 251.

(57) Benziger, J.; Preston, R. E. J. Phys. Chem. 1985, 89, 5002.

(58) Albert, M. R.; Lu, J. P.; Bernasek, S. L.; Cameron, S. D.; Gland, J. L. Surf. Sci. 1988, 206, 348.

(59) Castro, M. E.; Ahkter, S.; Golchet, A.; White, J. M. Langmuir, 1991, 7, 126

(60) Hyntley, D. R. J. Phys. Chem. 1989, 93, 6156.

(61) Weast, R. C., Selby, S. M., Eds. Handbook of Chemistry and Physics; Chemical Rubber Co.: Cleveland, OH, 1967. 